\title{
Repairing rabbit radial defects by combining bone marrow stroma stem cells with bone scaffold material comprising a core-cladding structure
}

\author{
H. Wu ${ }^{1,2}$, G.H. Liu ${ }^{2}$, Q. $W u^{1}$ and B. $Y u^{1}$ \\ ${ }^{1}$ Southern Medical University, Guangzhou, China \\ ${ }^{2}$ Second Affiliated Hospital of Inner Mongolia Medical University, Hohhot, China \\ Corresponding author: B. Yu \\ E-mail: yubinsmu@163.com
}

Genet. Mol. Res. 14 (4): 11933-11943 (2015)

Received January 8, 2015

Accepted May 18, 2015

Published October 5, 2015

DOI http://dx.doi.org/10.4238/2015.October.5.7

ABSTRACT. We prepared a bone scaffold material comprising a PLGA/ $\beta$ TCP core and a Type I collagen cladding, and recombined it with bone marrow stroma stem cells (BMSCs) to evaluate its potential for use in bone tissue engineering by in vivo and in vitro experiments. PLGA/ $\beta$ TCP without a cladding was used for comparison. The adherence rate of the BMSCs to the scaffold was determined by cell counting. Cell proliferation rate was determined by the 3-(4,5-dimethylthiazol-2-yl)-2,5diphenyltetrazolium bromide method. The osteogenic capability was evaluated by alkaline phosphatase activity. The scaffold materials were recombined with the BMSCs and implanted into a large segmental rabbit radial defect model to evaluate defect repair. Osteogenesis was assessed in the scaffold materials by histological and double immunofluorescence labeling, etc. The adherence number, proliferation number, and alkaline phosphatase expression of the cells on the bone scaffold material with core-cladding structure were significantly higher than the corresponding values in the PLGA/ $\beta$-TCP composite scaffold material $(P<0.05)$. An 
in vivo test indicated that the bone scaffold material with core-cladding structure completely degraded at the bone defect site and bone formation was completed. The rabbit large sentimental radial defect was successfully repaired. The degradation and osteogenesis rates matched well. The bone scaffold with core-cladding structure exhibited better osteogenic activity and capacity to repair a large segmental bone defect compared to the PLGA/ $\beta$-TCP composite scaffold. The bone scaffold with core-cladding structure has excellent physical properties and biocompatibility. It is an ideal scaffold material for bone tissue engineering.

Key words: Core-cladding structure; Osteogenic capability; Bone defect; Bone tissue engineering

\section{INTRODUCTION}

In tissue engineering, autologous, high-concentration, tissue-engineered seed cells are separated and seeded to a natural or synthetic cell scaffold or an extracellular matrix following in vitro culture for amplification (Sreejit and Verma, 2013). These biomaterial scaffolds must have excellent biocompatibility, and be capable of degradation and absorption by the human body. They can provide a three-dimensional space for cell survival and help cells obtain sufficient nutrients (Varoni et al., 2012). The biomaterial scaffold combined with cells can be implanted at the site of a bone defect. The seeded cells proliferate continuously and differentiate into osteoblasts, thus repairing osseous tissue defects while the biomaterial scaffold gradually degrades (Fassina et al., 2010). With the numerous breakthroughs in bone tissue engineering, this method has gradually become the most promising approach to the treatment of large segmental bone defects.

The PLGA/ $\beta$-TCP composite material basically meets the requirements of an ideal bone tissue engineering scaffold, i.e., it has excellent processing and molding characteristics, a high void ratio, excellent mechanical strength, and an appropriate degradation rate (Shahini et al., 2014). However, the strong hydrophobicity of its surface is detrimental to cellular adhesion, proliferation, and osteogenic differentiation, and substantially affects its repair capacity as a scaffold material. Research has demonstrated that the hydrophilicity of a porous scaffold material is effectively improved after it is covered with a layer of collagen (Tsai et al., 2014). Based on the considerations listed above, we established a bionic, bone tissue-engineered osteogenesis repair strategy using a low temperature deposition technique. We developed a highly bionic, complex, three-dimensional bone scaffold interior comprising PLGA/ $\beta$-TCP and an outer Type I collagen core-cladding structure to improve the hydrophilicity and biocompatibility of the bone scaffold material.

\section{MATERIAL AND METHODS}

\section{Materials}

Dulbecco's modified Eagle's medium and fetal bovine serum were purchased from Yanjing Biotechnology (Shanghai, China). The trypsin and osteogenesis induction solution were purchased from the Amresco Company (Albany, NY, USA). The $p$-nitrophenol was purchased from the Invitrogen Company (Carlsbad, CA, USA). The $\mathrm{CO}_{2}$ incubator was purchased from South-North Apparatus (Zhengzhou, China). The centrifuge was purchased from Jingli Centrifuge (Beijing, China) The inverted microscope was purchased from the MEDICA Company (Santa Clara, CA, USA). 


\section{Culture and passaging of primary cells}

A New Zealand white rabbit aged 1 day was sacrificed by decapitation, and immersed in $75 \%$ alcohol for $30 \mathrm{~min}$. The skin of the rabbit limbs was cut open aseptically to separate the muscles and completely remove the long bones from the limbs. Both ends of the long bones were sheared. The middle part of each remaining long bone was immediately placed in Dulbecco's modified Eagle's medium containing $10 \%$ fetal bovine serum. An appropriate amount of culture solution was extracted with a 2-mL syringe to repeatedly wash the marrow cavities of the long bones. The rat bone marrow cells were cultured and passaged according to the procedure described in the literature (Liu et al., 2013).

\section{Inoculating cells into the scaffold material}

The culture solution on the immersed surface of the scaffold material was absorbed by sterile filter paper. The scaffold material was then placed on a sterile 6-well plate. The prepared cell suspension $(10 \mu \mathrm{L})$ was then gently pipetted onto the surface of the scaffold material. After it had permeated through the scaffold material completely, an additional cell suspension $(10 \mu \mathrm{L})$ was added dropwise until the whole scaffold material had been completely permeated by the cell suspension. The scaffold material inoculated with cells was placed in a cell incubator and carefully transferred to another sterile 6-well plate after $6 \mathrm{~h}$. An appropriate amount of cell culture solution was added for continuous culture. The culture solution was replaced every 3 days (Walser et al., 2013).

\section{Determination of adhesive capacity of cells on the scaffold material}

A total of $2 \times 10^{5}$ cells were seeded on each sample material. The scaffold materials were taken out after co-culture for 2, 4, and $6 \mathrm{~h}$, and washed with phosphate-buffered saline (PBS). After complete digestion, the cell solution was placed in a centrifuge tube and centrifuged for 5 min at $1000 \mathrm{rpm}$. The supernatant was discarded. The cells were resuspended with an appropriate amount of cell culture solution. The resuspended solution was stained with $0.4 \%$ trypan blue solution at a volume ratio of 1:1. A globulimeter was used for counting under a phase contrast optical microscope (Sadiasa et al., 2014).

\section{Determination of proliferation capacity of cells on the scaffold material}

The proliferation capacity of the bone marrow stroma stem cells (BMSCs) was determined using 3-(4,5-dimethylthiazol-2-yl)-2,5-diphenyltetrazolium bromide (MTT). Cells were seeded on each sample material at a cell concentration of $1 \times 10^{7}$ cells $/ \mathrm{mL}$. The scaffold materials were taken out after co-culture for 3, 6, 9, and 12 days, and washed three times with PBS to remove the cells not effectively adhered to their surfaces. The optical density (OD) values were measured at 490 $\mathrm{nm}$. The number of cells was measured using the MTT method.

\section{Determination of osteogenic differentiation capacity of cells on the scaffold material (alkaline phosphatase method)}

The BMSCs and the scaffold materials were combined. They were then placed in the osteogenesis induction solution. The scaffold materials co-cultured with the cells were taken out 
from the culture plate at 6,9 , and 12 days, and washed gently three times with PBS. Glycine solution (0.1 M, pH 13.0) containing $0.1 \mathrm{~mL} p$-nitrobenzophenone was added to the cell suspension $(0.1$ $\mathrm{mL}$ ) after the cells had been separated by sonic degradation. The mixture was placed on a shaking table to interact for $30 \mathrm{~min}$ at $37^{\circ} \mathrm{C}$, then $0.3 \mathrm{~mL} 0.25 \mathrm{~N} \mathrm{NaOH}$ was immediately used to terminate the reaction. The absorbance value, which represents the content of alkaline phosphatase, was determined by measuring the emission of $p$-nitrophenol. The BMSCs without a scaffold material served as a blank control (Vishnoi and Kumar, 2013).

\section{Animal experiments}

The experimental animals comprised 45 healthy male or female New Zealand white rabbits, aged 3 months, weighing $2-3 \mathrm{~kg}$, with mature skeletal development. The rabbits were randomly divided into three groups: the bone scaffold material with core-cladding structure combined with BMSCs was implanted in Group A; the PLGA/ $\beta$-TCP composite scaffold material combined with BMSCs was implanted in Group B; and no scaffold material was implanted in Group C, which served as the blank control group.

\section{Establishment of rabbit radial defect models and implantation of the scaffold materials}

A skin preparation was made for the bilateral forelimbs of the rabbits. Sodium pentobarbital $(3 \%)$ was injected intramuscularly $(0.6 \mathrm{~mL} / \mathrm{kg})$. The rabbit was fixed onto the operating floor after successful anesthetization. A longitudinal incision was made on the radialis side of the forearm. The skin and subcutaneous fascia layer were cut open in sequence. Blunt dissection was performed for the radialis lateral muscle to expose the proximal end of the radius and strip the periosteum. The radius was cut off at $2-3 \mathrm{~cm}$ and $4.5-5.5 \mathrm{~cm}$ from the capitulum of the radius with a dental driller. A 1.5-cm long bone segment was removed to create a large segmental rabbit bone defect model. The different scaffold materials were implanted according to the different groups after hemostasis and irrigation. No internal fixation was performed after implantation. The intermuscular space was sutured. The scaffold material covered the bone defect. Sutures were performed layer by layer. Gentamicin $(400,000 \mathrm{U})$ was intramuscularly injected into each rabbit per day for 3 consecutive days after operation. The animals were sacrificed at 12, 24, and 48 weeks after operation. Five animals from each group were sacrificed each time (Zou et al., 2014).

The rabbits were intramuscularly injected with tetracycline for 2 consecutive days at a dose of $50 \mathrm{mg} / \mathrm{kg} 14$ days before the time of material selection at 24 weeks. The rabbits were intramuscularly injected with calcein for 2 consecutive days at a dose of $8 \mathrm{mg} / \mathrm{kg} 3$ days before the time of material selection at 24 weeks.

\section{Tetracycline-calcein double immunofluorescence labeling observation}

The animals were sacrificed at 24 weeks after operation for material selection. The material was fixed with $80 \%$ alcohol, placed in a dehydrator, embedded in plastic, sectioned, and investigated using double immunofluorescence labeling.

\section{Statistical analysis}

All data are reported as means \pm standard deviation. The SPSS 13.0 software was used 
for statistical treatment. A $t$-test was conducted for comparisons among groups. Differences were considered statistically significant when $\mathrm{P}<0.05$.

\section{RESULTS}

\section{Morphological observation of BMSCs}

The primary cells did not differentiate. The cells were circular or irregularly polygonal. The cell nuclei were circular or oval. Generation II cells gradually separated from the colony center. They grew in a radial or whirl pattern, and exhibited fibroblast-like morphology. Generation III cells gradually concentrated in the colony center. They also grew in a radial or whirl pattern, and exhibited a fibroblast-like morphology. The cells were distributed like paving stones at 3 weeks after induction. Circular or oval calcified nodules were present in the cell community. The cells around the nodules were distributed in a radial pattern, and were large. The cell nuclei were distributed in the center of the cytoplasm. The proportion of nucleoplasm was large. The cells resembled the morphological expression of osteoblasts (Figure 1).
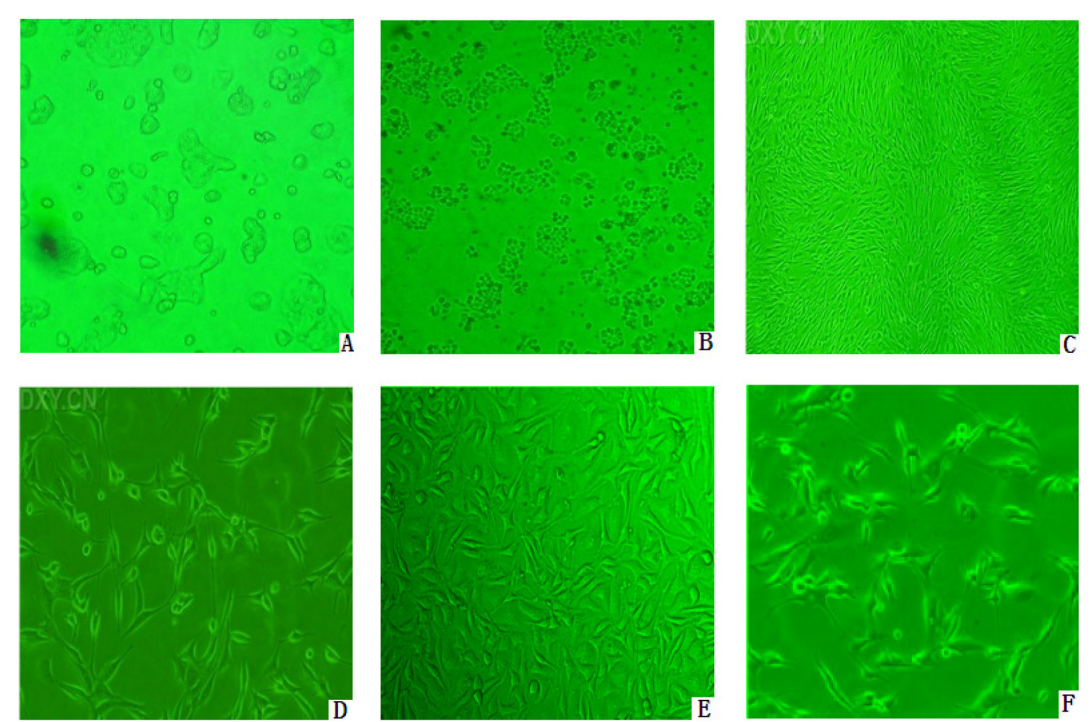

Figure 1. Morphological comparison of bone marrow stroma stem cells. A. Morphology of primary cells; B. morphology of Generation I cells; C. morphology of Generation II cells; D. morphology of Generation III cells; E. morphology of cells 1 week after osteogenesis induction; F. morphology of cells 3 weeks after osteogenesis induction.

\section{Determination of adhesive capacity of cells on the scaffold material}

The results of the cell counting experiment indicated that the number of cells adhered to the scaffold materials in both the relevant groups increased significantly $(P<0.05)$ at $2-4 \mathrm{~h}$ after co-culture of the cells and scaffold materials. There was no statistically significant difference in the number of cells adhered to the scaffold materials between the groups at 4-6 $\mathrm{h}$. There were significantly more cells adhered to the bone scaffold material with core-cladding structure than to the PLGA/ $\beta-T C P$ composite scaffold material at 2-6 h $(P<0.05)$ (Figure 2). 


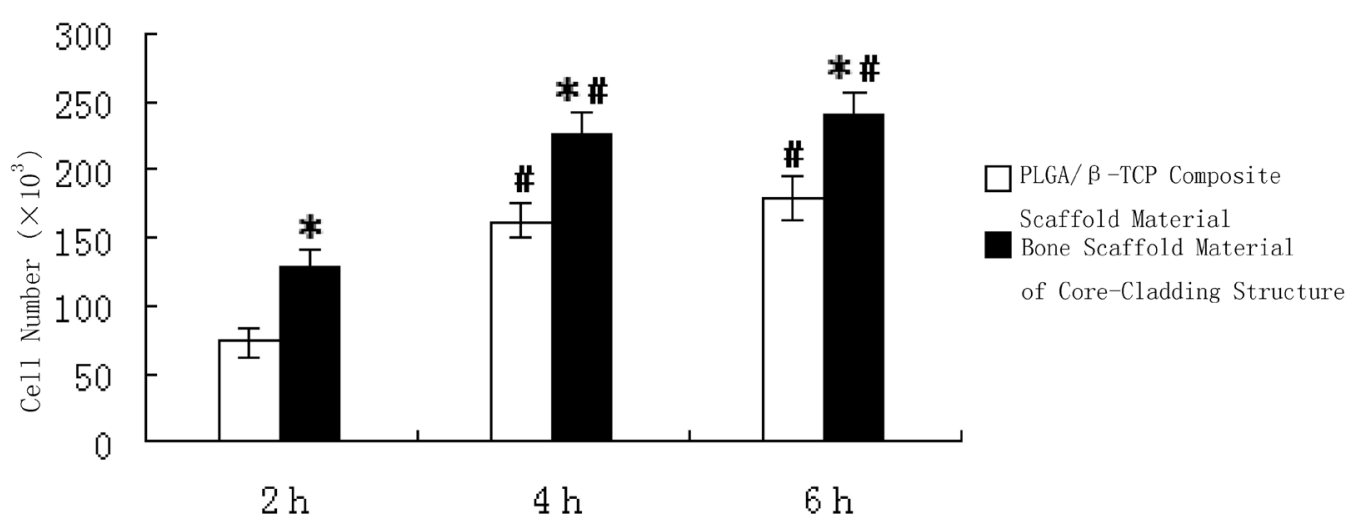

Figure 2. Results of analysis of cell adhesion to the scaffold material in both relevant groups. Compared with the PLGA/ $\beta$-TCP composite scaffold material group, ${ }^{*} \mathrm{P}<0.05$; compared with co-culture for $2 \mathrm{~h},{ }^{\#} \mathrm{P}<0.05$.

\section{Determination of proliferation capacity of cells on the scaffold material}

The MTT experimental results showed that the number of cells on the scaffold materials in both relevant groups increased significantly at 3-12 days after co-culture of cells and scaffold materials $(P<0.05)$; the number of the cells on the bone scaffold material with core-cladding structure was significantly higher than that on the PLGA/ $\beta$-TCP composite scaffold material from the 3rd day $(P<0.05)$ (Figure 3$)$.

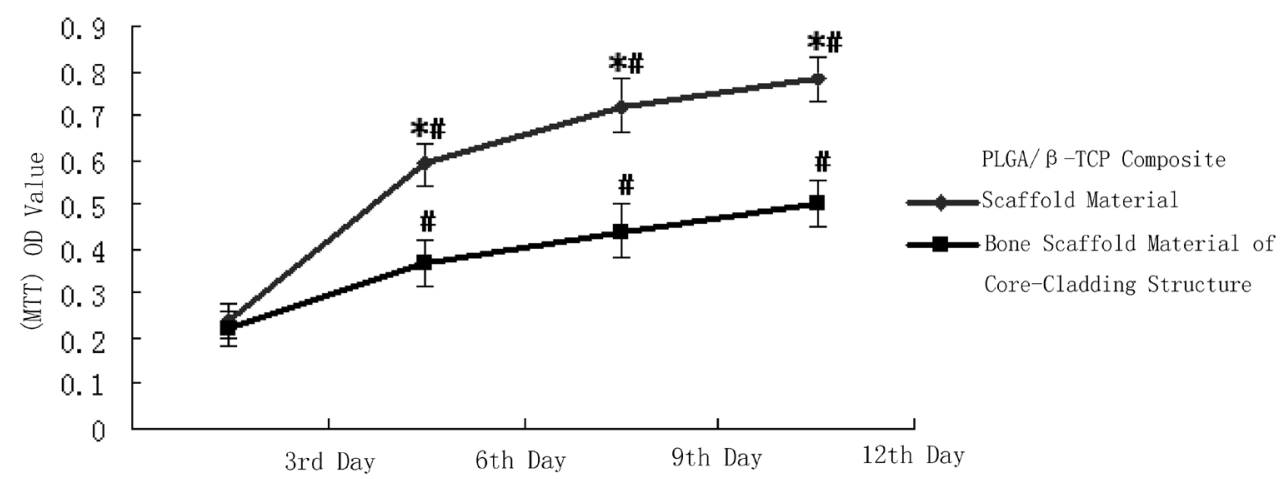

Figure 3. Results of analysis of cell proliferation on the scaffold material in both relevant groups. Compared with the PLGA/ $\beta$-TCP composite scaffold material group, ${ }^{*} \mathrm{P}<0.05$; compared with 3 days after co-culture, $\mathrm{P} P<0.05$. MTT = 3-(4,5-dimethylthiazol-2-yl)-2,5-diphenyltetrazolium bromide; OD = optical density.

\section{Determination of osteogenesis differentiation capacity of cells on the scaffold material}

The experimental results showed that the expression of alkaline phosphatase in cells on the scaffold materials in both relevant groups increased slightly at 6-14 days after co-culture of cells and scaffold materials; the expression of alkaline phosphatase in the cells on the bone scaffold material with core-cladding structure was significantly higher than that in the cells on the PLGA/ $\beta$ TCP composite scaffold material $(P<0.05)$ (Figure 4). 


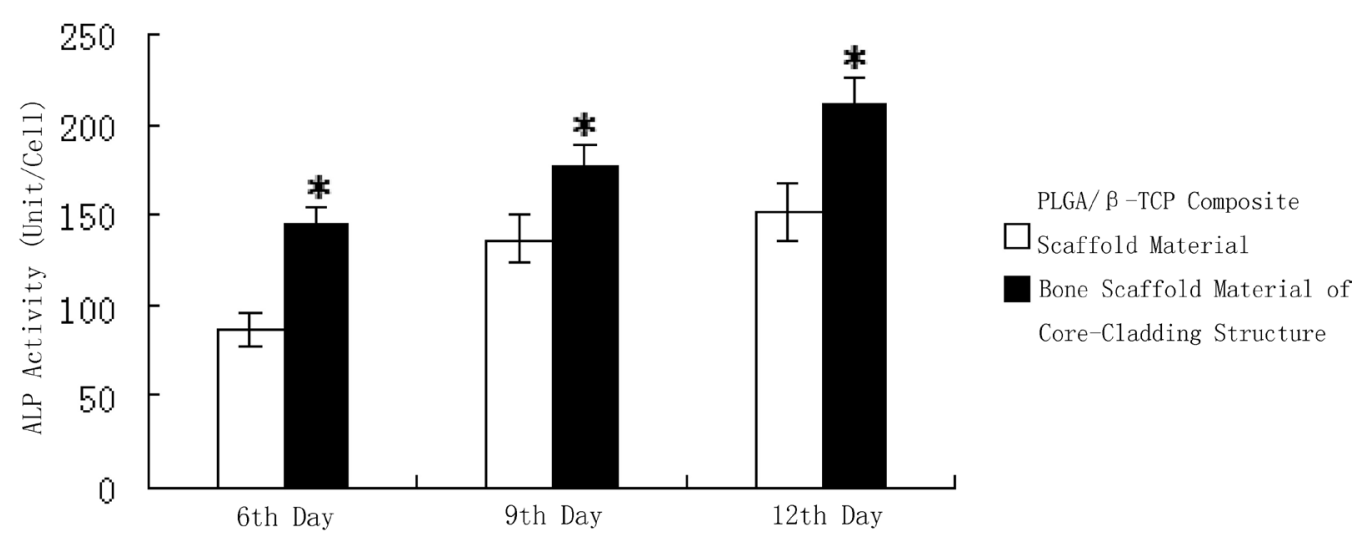

Figure 4. Results of quantitative analyses of alkaline phosphatase (ALP) activity in the scaffold material in both relevant groups. Compared with the PLGA/ $\beta$-TCP composite scaffold material group, ${ }^{*} \mathrm{P}<0.05$.

\section{Histomorphological results of animal experiments}

At 12 weeks after operation, the scaffold material in Group A had degraded partially and woven bones were present on the surface; the scaffold material in Group B had degraded insignificantly with less formation of woven bones. At 24 weeks after operation, the scaffold material in Group A had almost completely degraded and had been substituted by woven bone; the medullary canal had a tendency of repatency; the scaffold material in Group B had degraded significantly and formation of woven bones had increased. At 48 weeks after operation, the scaffold material in Group A had degraded completely; the medullary canal was once more unobstructed; bone molding had been completed; the scaffold material in Group B had completely degraded and the medullary canal was occluded, as shown in Figure 5.
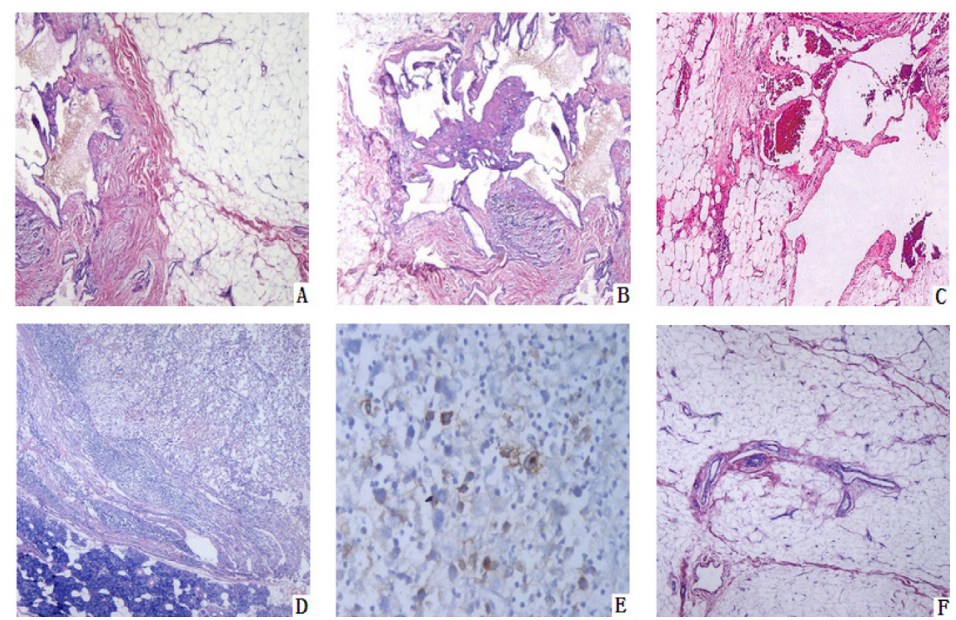

Figure 5. Results of hematoxylin and eosin (H\&E) staining. A. H\&E staining at 12 weeks after operation in Group A; B. H\&E staining at 24 weeks after operation in Group A; C. H\&E staining at 36 weeks after operation in Group A; D. H\&E staining at 12 weeks after operation in Group B; E. H\&E staining at 24 weeks after operation in Group B; F. H\&E staining at 36 weeks after operation in Group B. 


\section{Tetracycline-calcein double immunofluorescence labeling results}

The double immunofluorescence labeling results of the specimens sampled at 24 weeks after operation indicated that the double immunofluorescence labeling area in Group A was dense compared with Group B, as shown in Figure 6.
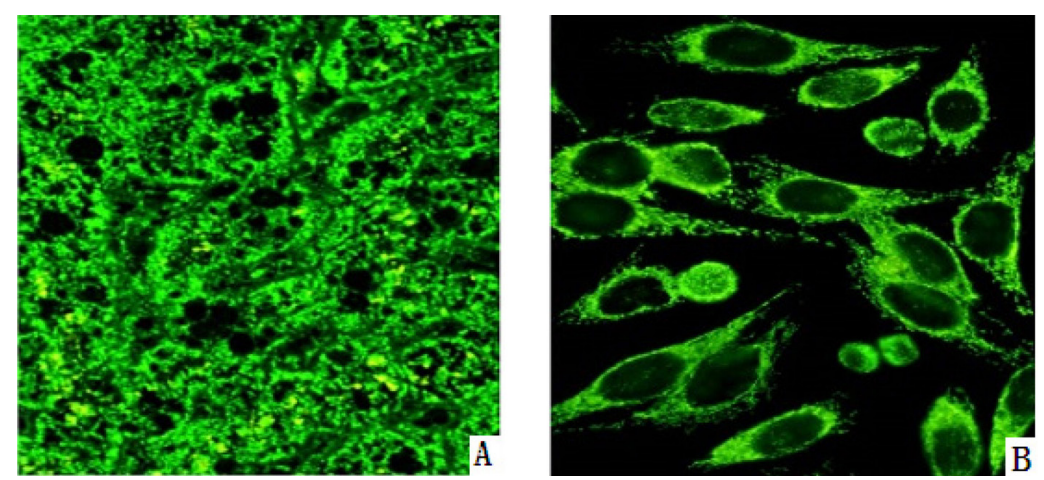

Figure 6. Results of tetracycline-calcein double immunofluorescence labeling. A. Tetracycline-calcein double immunofluorescence labeling result in Group A 24 weeks after operation; B. tetracycline-calcein double immunofluorescence labeling result in Group B 24 weeks after operation.

\section{DISCUSSION}

Orthopedic diseases that cause large segmental bone defects, such as severe trauma, fractures in osteoporosis patients, tumors, and congenital malformation of the musculoskeletal system, are very common (Feng et al., 2013). To treat these diseases, the affected bone must be excised, leading to large segmental bone defects. Reparation of bone defects is the greatest treatment challenge (Sajesh et al., 2013). With numerous breakthroughs in recent years, bone tissue engineering has gradually become the most promising method for the treatment of large segmental bone defects. Bone tissue engineering primarily consists of three major factors. The bone scaffold material is the most critical factor in bone tissue engineering because it serves as a carrier of seed cells and active factors, and provides support for the formation of new bones (Kang and Cho, 2012; Paşcu et al., 2013). Proper structure, appropriate physical properties, and excellent biocompatibility are the most important requirements of an ideal bone scaffold material. As a temporary supporting structure, the bone scaffold material determines the final shape of the new bone and affects the interaction among cells that combine with the scaffold material. The cells growing on the surface of the scaffold material exhibit different biological activities as a result of differences in hydrophilicity, roughness, etc. (Liundup et al., 2013; Murphy et al., 2013).

It is well known that the surface of a scaffold material is the first point of contact and interaction for the cells. Therefore, the surface characteristics of the scaffold material have a great effect on a series of cellular reactions, such as adhesion, proliferation, and differentiation (Rao and Stegemann, 2013). A great deal of research has demonstrated that the extracellular matrix plays an important role in cell adhesion, proliferation, and osteogenic differentiation. Establishing extracellular matrices in vitro enables the cells to easily adhere to the surface of a scaffold material (Lu et al., 2013; Moshaverinia et al., 2013). Research has also demonstrated that introducing short peptides that promote cell adhesion, such as fibronectin, Asp-Gly-Glu-Ala (DGEA), and Arg-Gly- 
Asp (RGD) sequences, to the surface of the matrix lining by solvent linking or lining can effectively improve the biological activity of cells and promote their adhesion to the surface of the scaffold material (Asaoka et al., 2013). Type I collagen not only contains many collagen molecules of major components of the extracellular matrix but also has good hydrophilicity and low immunogenicity (Dias et al., 2012). Thus, we chose to use Type I collagen in the core-cladding structure to improve the hydrophilicity of the scaffold material.

Bone tissue engineering has two critical factors: scaffold materials and seed cells. As a temporary support structure, the scaffold material not only affects interactions among the cells combining with it, but also ultimately determines the shape of the new bones. Thus, it is important that the scaffold material provides an environment that contributes to rapid growth, proliferation, and osteogenic differentiation, and ensures the generation of new bone tissue (Amini et al., 2012; Cai et al., 2012). The seed cells need to proliferate and differentiate to osteoblasts rapidly, thus promoting generation of new bone tissue. As a glycoprotein on the cell surface, alkaline phosphatase acts as a marker indicating the differentiation of cells and osteogenesis (Delabarde et al., 2012).

Here, the experimental results showed that the BMSCs combined with the bone scaffold material with core-cladding structure and exhibited a higher alkaline phosphatase activity compared with the BMSCs combined with the PLGA/ $\beta$-TCP composite scaffold material. This indicates that the number of BMSCs that combined with the core-cladding scaffold material and differentiated to osteoblasts was higher, demonstrating that the core-cladding scaffold structure has a greater capacity to induce osteogenic differentiation and exhibits greater biocompatibility.

The adhesion of seed cells to the surface of the scaffold material significantly affects the cellular morphology, as well as further proliferation and osteogenic differentiation ( $\mathrm{He}$ et al., 2012). The process of adhesion of seed cells to the scaffold material is very complex and is influenced by various factors. The surface characteristics of the scaffold material, such as its electrical characteristics, free energy, and hydrophilicity, play a decisive role in the adhesion of the seed cells because contact between the seed cells and the scaffold material takes place at the scaffold surface (Jakob et al., 2012; Nguyen et al., 2012). Research has demonstrated that high surface hydrophilicity of a scaffold material can effectively improve the adhesion of the seed cells (Tarafder et al., 2013). Here, the experimental results indicated that the surface hydrophilicity of the bone scaffold material with core-cladding structure improved considerably compared with the PLGA/ $\beta$-TCP composite scaffold material. This may be because the core-cladding scaffold exhibits better cell adhesion, proliferation, and osteogenic differentiation capabilities. The reason for the considerable improvement in the surface hydrophilicity of the scaffold material with corecladding structure is that it was covered with Type I collagen. Moreover, the experimental results demonstrated that the core-cladding scaffold material had greater biocompatibility.

Based on the principles of bionics, we simulated the authentic structure of human bone tissue, established a repair strategy in combination with a low-temperature deposition technique, and developed a controllable annular cannula nozzle for the experiment. We made a complex, highly bionic, three-dimensional bone core-cladding material with a structure appropriate for cellular distribution to improve the hydrophilicity and biocompatibility of the bone scaffold. The in vitro experiment demonstrated that the surface of the core-cladding bone scaffold material had significantly improved hydrophilicity. Moreover, the BMSCs exhibited better adhesion, proliferation, and osteogenic differentiation capabilities on the surface of the core-cladding bone scaffold material, indicating that it had greater biocompatibility. As the main protein of the extracellular matrix, Type I collagen contains specific cell binding sites on its surface that contribute to cell adhesion and growth of new blood vessels. These directly affect the generation and remolding of 
the new bone tissue (Bellucci et al., 2012; Wen et al., 2013). The experimental results sufficiently demonstrated that the core-cladding bone scaffold material had better osteogenic activity and degradability, and could repair a rabbit radial defect more quickly, compared with conventional scaffold structures. Based on histological observations, we discovered that the degradation rate of the core-cladding bone scaffold material matched the formation of new osseous tissue. The double immunofluorescence labeling observations of the specimens at 24 weeks revealed that the area of the double immunofluorescence labeling in Group A was significantly larger than that in Group B, and the distance between the fluorescence bands was large, indicating that the osteogenesis area on the scaffold material was more extensive, the number of new bones formed was higher, and the osteogenic capability was stronger in Group A than in Group B at 14 days before the sampling time, which was 24 weeks after operation.

\section{Conflicts of interest}

The authors declare no conflict of interest.

\section{REFERENCES}

Amini AR, Laurencin CT and Nukavarapu SP (2012). Differential analysis of peripheral blood- and bone marrow-derived endothelial progenitor cells for enhanced vascularization in bone tissue engineering. J. Orthop. Res. 30: 1507-1515.

Asaoka T, Ohtake S, Furukawa KS, Tamura A, et al. (2013). Development of bioactive porous $\alpha$-TCP/HAp beads for bone tissue engineering. J. Biomed. Mater. Res. A. 101: 3295-3300.

Bellucci D, Chiellini F, Ciardelli G, Gazzarri M, et al. (2012). Processing and characterization of innovative scaffolds for bone tissue engineering. J. Mater. Sci. Mater. Med. 23: 1397-1409.

Cai YZ, Zhang GR, Wang LL, Jiang YZ, et al. (2012). Novel biodegradable three-dimensional macroporous scaffold using aligned electrospun nanofibrous yarns for bone tissue engineering. J. Biomed. Mater. Res. A. 100: 1187-1194.

Delabarde C, Plummer CJ, Bourban PE and Månson JA (2012). Biodegradable polylactide/hydroxyapatite nanocomposite foam scaffolds for bone tissue engineering applications. J. Mater. Sci. Mater. Med. 23: 1371-1385.

Dias MR, Fernandes PR, Guedes JM and Hollister SJ (2012). Permeability analysis of scaffolds for bone tissue engineering J. Biomech. 45: 938-944.

Fassina L, Saino E, Visai L, Avanzini MA, et al. (2010). Use of a gelatin cryogel as biomaterial scaffold in the differentiation process of human bone marrow stromal cells. Conf. Proc. IEEE Eng. Med. Biol. Soc. 2010: 247-250.

Feng L, Wu H, E L, Wang D, et al. (2013). Effects of vascular endothelial growth factor 165 on bone tissue engineering. PLoS One 8: e82945.

He N, Elingarami S and Zhu P (2012). Application of solid-state NMR in characterization of bone related tissue engineering. J. Nanosci. Nanotechnol. 12: 2858-2865.

Jakob M, Saxer F, Scotti C, Schreiner S, et al. (2012). Perspective on the evolution of cell-based bone tissue engineering strategies. Eur. Surg. Res. 49: 1-7.

Kang HW and Cho DW (2012). Development of an indirect stereolithography technology for scaffold fabrication with a wide range of biomaterial selectivity. Tissue Eng. Part C Methods 18: 719-729.

Liu M, Yu X, Huang F, Cen S, et al. (2013). Tissue engineering stratified scaffolds for articular cartilage and subchondral bone defects repair. Orthopedics 36: 868-873.

Liundup AV, Medvedev luA, Balasanova KV, Zolotopup NM, et al. (2013). [Methods of tissue engineering of bone tissue in maxillofacial surgery]. Vestn. Ross. Akad. Med. Nauk. 5: 10-5.

Lu CH, Chang YH, Lin SY, Li KC, et al. (2013). Recent progresses in gene delivery-based bone tissue engineering. Biotechnol. Adv. 31: 1695-1706.

Moshaverinia A, Chen C, Akiyama K, Xu X, et al. (2013). Encapsulated dental-derived mesenchymal stem cells in an injectable and biodegradable scaffold for applications in bone tissue engineering. J. Biomed. Mater. Res. A. 101: 3285-3294.

Murphy CM, O'Brien FJ, Little DG, Schindeler A, et al. (2013). Cell-scaffold interactions in the bone tissue engineering triad. Eur. Cell Mater. 26: 120-132.

Nguyen LH, Annabi N, Nikkhah M, Bae H, et al. (2012). Vascularized bone tissue engineering: approaches for potential improvement. Tissue Eng. Part B Rev. 18: 363-382. 
Paşcu El, Stokes J and McGuinness GB (2013). Electrospun composites of PHBV, silk fibroin and nano-hydroxyapatite for bone tissue engineering. Mater. Sci. Eng. C. Mater. Biol. Appl. 33: 4905-4916.

Rao RR and Stegemann JP (2013). Cell-based approaches to the engineering of vascularized bone tissue. Cytotherapy 15: 1309-1322.

Sadiasa A, Nguyen TH and Lee BT (2014). In vitro and in vivo evaluation of porous PCL-PLLA 3D polymer scaffolds fabricated via salt leaching method for bone tissue engineering applications. J. Biomater. Sci. Polym. Ed. 25: 150-167.

Sajesh KM, Jayakumar R, Nair SV and Chennazhi KP (2013). Biocompatible conducting chitosan/polypyrrole-alginate composite scaffold for bone tissue engineering. Int. J. Biol. Macromol. 62: 465-471.

Shahini A, Yazdimamaghani M, Walker KJ, Eastman MA, et al. (2014). 3D conductive nanocomposite scaffold for bone tissue engineering. Int. J. Nanomedicine 9: 167-181.

Sreejit P and Verma RS (2013). Natural ECM as biomaterial for scaffold based cardiac regeneration using adult bone marrow derived stem cells. Stem Cell Rev. 9: 158-171.

Tarafder S, Balla VK, Davies NM, Bandyopadhyay A, et al. (2013). Microwave-sintered 3D printed tricalcium phosphate scaffolds for bone tissue engineering. J. Tissue Eng. Regen. Med. 7: 631-641.

Tsai WB, Chen WT, Chien HW, Kuo WH, et al. (2014). Poly(dopamine) coating to biodegradable polymers for bone tissue engineering. J. Biomater. Appl. 28: 837-848.

Varoni E, Tschon M, Palazzo B, Nitti P, et al. (2012). Agarose gel as biomaterial or scaffold for implantation surgery: characterization, histological and histomorphometric study on soft tissue response. Connect. Tissue Res. 53: 548-554.

Vishnoi T and Kumar A (2013). Conducting cryogel scaffold as a potential biomaterial for cell stimulation and proliferation. J. Mater. Sci. Mater. Med. 24: 447-459.

Walser R, Metzger W, Görg A, Pohlemann T, et al. (2013). Generation of co-culture spheroids as vascularisation units for bone tissue engineering. Eur. Cell Mater. 26: 222-233.

Wen Y, Jiang B, Cui J, Li G, et al. (2013). Superior osteogenic capacity of different mesenchymal stem cells for bone tissue engineering. Oral Surg. Oral Med. Oral Pathol. Oral Radiol. 116: e324-e332.

Zou J, Yuan C, Wu C, Cao C, et al. (2014). Isolation and osteogenic differentiation of skeletal muscle-derived stem cells for bone tissue engineering. Mol. Med. Rep. 9: 185-191. 